\title{
Vascular dysfunction measured by fingertip thermal monitoring is associated with the extent of myocardial perfusion defect
}

\author{
Naser Ahmadi, MD, ${ }^{\mathrm{a}}$ Nudrat Usman, $M D,{ }^{\mathrm{a}}$ John Shim, MD, ${ }^{\text {a }}$ Vivek Nuguri, MD, ${ }^{\mathrm{a}}$ \\ Panukorn Vasinrapee, MD, ${ }^{\text {a }}$ Fereshteh Hajsadeghi, MD, ${ }^{\mathrm{a}}$ Zhiying Wang, MS, ${ }^{\mathrm{b}}$ \\ Gary P. Foster, $M D,{ }^{c}$ Khurram Nasir, MD, MPH, ${ }^{\mathrm{d}}$ Harvey Hecht, $M D,{ }^{\mathrm{e}}$ \\ Morteza Naghavi, $M D{ }^{d}$ and Matthew Budoff, $M D^{a}$
}

Background. Previous studies have shown that vascular dysfunction measured by digital thermal monitoring (DTM) during an arm-cuff reactive hyperemia procedure correlates with the severity of coronary artery disease measured by coronary artery calcium in asymptomatic patients. Current study investigates the correlation between DTM and abnormal myocardial perfusion imaging (MPI).

Methods. About 116 consecutive patients with chest discomfort, age $57 \pm 10$ years, underwent MPI, DTM and Framingham Risk Score (FRS) assessment. Fingertip temperature rebound (TR), DTM index of vascular reactivity, was assessed after a 2-minute arm-cuff reactive hyperemia test. The extent of myocardial perfusion defect was measured by summed stress score (SSS).

Results. TR decreased from SSS $<4(1.61 \pm 0.15)$ to $4 \leq \mathrm{SSS} \leq 8(0.5 \pm 0.22)$ to $9 \leq \mathrm{SSS} \leq 13(0.26 \pm 0.15)$ to SSS $>13(-0.37 \pm 0.19)(P=.0001)$. After adjusting for cardiac risk factors, the odds ratio of the lowest versus two upper tertiles of TR was 3.93 for SSS $\geq 4$ and 9.65 for SSS $\geq 8$ compared to SSS $<4$. TR correlated well with SSS $(r=-0.88, P=.0001)$. Addition of TR to FRS increased the area under the ROC curve to predict abnormal MPI, SSS $\geq 4$, from 0.65 to $0.84(P<.05)$.

Conclusion. Vascular dysfunction measured by DTM is associated with the extent of myocardial perfusion defect independent of age, gender, and cardiac risk factors. ( $J$ Nucl Cardiol 2009;16:431-9.)

Key Words: Myocardial perfusion defect $\cdot$ myocardial perfusion imaging $\cdot$ digital thermal monitoring

The role of vascular dysfunction in cardiovascular disease is well established ${ }^{1-5}$ and has been documented

Electronic supplementary material The online version of this article (doi:10.1007/s12350-008-9044-y) contains supplementary material, which is available to authorized users.

From Los Angeles Biomedical Research Institute, ${ }^{\text {a }}$ Harbor UCLA Medical Center, Torrance, CA; University of Texas, ${ }^{\mathrm{b}}$ Houston; VA Loma Linda Health Care System, ${ }^{c}$ Loma Linda University School of Medicine, Loma Linda, CA; Society for Heart Attack Prevention and Eradication, ${ }^{\mathrm{d}}$ Houston, TX and Lenox Hill Heart \& Vascular Institute, ${ }^{\mathrm{e}}$ New York, NY.

Received for publication Jul 7, 2008; final revision accepted Dec 7, 2008.

Reprint requests: Matthew Budoff, MD, Los Angeles Biomedical Research Institute, Harbor UCLA Medical Center, 1124 W. Carson Street, RB2, Torrance, CA 90502; mbudoff@labiomed.org.

$1071-3581 / \$ 34.00$

Copyright $(\subset$ 2008. The Author(s). This article is published with open access at Springerlink.com

doi:10.1007/s12350-008-9044-y by several technologies that evaluate reactive hyperemia during and following arm cuff occlusion. Fingertip digital thermal monitoring (DTM) of vascular function during cuff occlusion is a new non-invasive, operatorindependent test based on changes in fingertip temperature during and after arm cuff occlusion. It has been shown to correlate with the severity of coronary artery disease in asymptomatic patients, measured by coronary artery calcium. ${ }^{6,7}$ The present study was designed to evaluate whether vascular dysfunction measured by DTM correlates with abnormal myocardial perfusion imaging (MPI) in symptomatic patients.

\section{METHODS}

\section{Subjects and Study Design}

The study population included 116 consecutive referral patients with suspected coronary artery disease (CAD) to the 
nuclear laboratory (45 with typical angina, 31 with atypical angina, 35 with shortness of breath, and 5 with known CAD). All subjects underwent myocardial perfusion imaging (MPI) and DTM. Subjects over 35 years were eligible to participate. Patients with diabetic retinopathy, peripheral vascular diseases, underlying infection, cancer, systemic inflammation, Raynaud's disease, immunosuppression, and end stage renal or liver disease were excluded. Cardiac risk factors, known coronary artery disease (CAD), including prior myocardial infarction (MI), angina, or intervention, were validated. Body mass index, hip circumference, blood pressure, fasting blood glucose, and lipid profile were obtained by standard techniques and the Framingham 10 year CAD risk score (FRS) was calculated. ${ }^{8}$

\section{Digital Thermal Monitoring of Vascular Function}

After an overnight fast and abstinence from tobacco, alcohol, caffeine, and vasoactive medications, the left arm blood pressure was recorded in a sitting position 15 minutes before the DTM test (Omron HEM 705 CP semi-automated sphygmomanometer, Bannockburn, IL, USA). After remaining at rest in a supine position in a room with temperature $22.5-25^{\circ} \mathrm{C}$ for 30 minutes, DTM of both hands was obtained during 3 minutes stabilization, 2 minutes cuff inflation to $50 \mathrm{mmHg}$ greater than systolic blood pressure, and 5 minutes deflation using an automated, operator-independent protocol (VENDYS6000, Endothelix Inc., Houston, TX). DTM thermal probes designed to minimize the area of skin probe contact and fingertip pressure were attached to the pulp of the index finger on both hands. Thermal changes before, during, and after armocclusive reactive hyperemia test were traced continuously in the fingertip of both occluded and non-occluded arm and digitalized automatically using VENDYS software (a computer based thermometry system with $0.006^{\circ} \mathrm{C}$ thermal resolution and an automated compressor for measurement of blood pressure and controlled occlusion hyperemia). The equations for postocclusive temperature rebound (TR) and area under the curve (AUC) are shown in Figure 1.

In a substudy, a fully automated DTM device (VENDYS, Endothelix Inc., Houston) was used for repeated measurement of vascular reactivity and endothelial function in 18 subjects

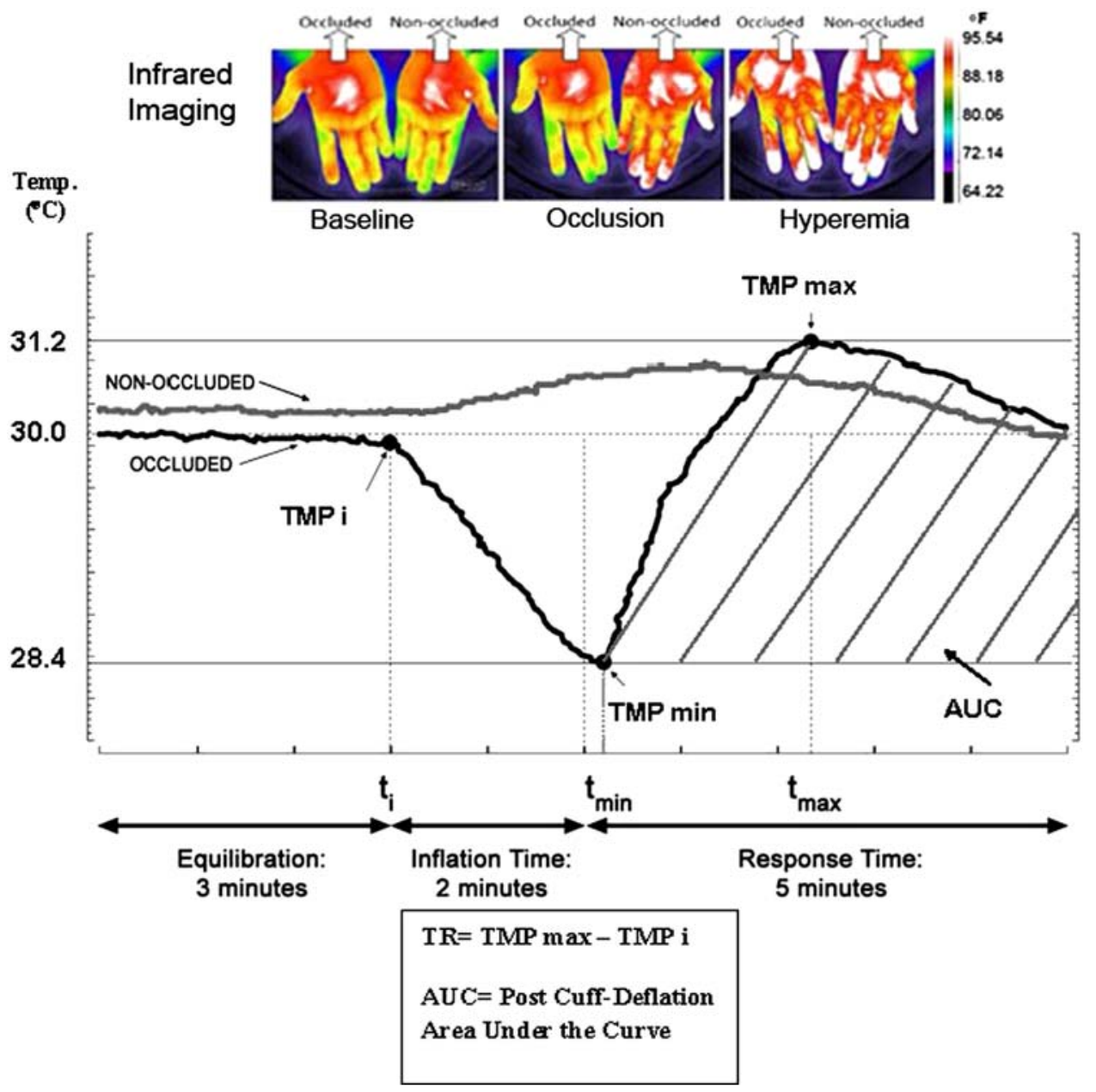

Figure 1. Skin temperature changes during cuff-reactive hyperemia, as shown by infrared imaging and digital thermal monitoring (DTM). 
without CAC (age $35 \pm 4$ years, 74\% male) in two tests performed 24 hours apart. All subjects underwent overnight fasting, and the test was preceded by a 30 minute rest in a supine position inside a dimmed room with temperature $22.5-25^{\circ} \mathrm{C}$. The measurements were obtained during and after a 5 minute supra systolic arm-cuff occlusion-induced reactive hyperemia. TR and AUC were studied as DTM indices of vascular function.

\section{SPECT Imaging}

Patients were instructed to fast and abstain from caffeineand methylxanthine-containing substances for 24 hours before testing. $370 \mathrm{MBq}$ Tc-99m sestamibi was injected at rest. After 45-60 minutes, single photon emission computed tomography (SPECT) was performed with a dual-head SPECT camera (SMV/ DST, ADAC/Vertex, Philips Medical Systems, Netherlands) with 64 total projections, $180^{\circ}$ right anterior oblique-left posterior oblique orbit, $64 \times 64$ matrix, $0.6-\mathrm{cm}$ pixel size, eight frames per cardiac cycle, low-energy, high-resolution collimation, and 30 seconds per stop. For the stress study, adenosine was infused at $140 \mu \mathrm{g} \cdot \mathrm{kg}^{-1} \cdot \min ^{-1}$ for 6 minutes. ${ }^{8,9}$ At the end of the third minute of the infusion, a weight-adjusted dose of Tc-99m sestamibi $(925 \mathrm{MBq}$ for $<102.2 \mathrm{~kg}$ and $1110 \mathrm{MBq}$ otherwise) was administered. After 30-45 minutes, patients underwent repeat imaging. The original scan images were processed and reconstructed using conventional vendor software and reviewed on both the computer workstation and on singleemulsion film. The initial clinical interpretation was provided by a pair of nuclear medicine specialists with extensive training and experience in cardiac nuclear medicine who blinded to the patient, treatment condition and DTM, jointly reviewed and reported each scan. At the time of the initial reading, the results were categorized as normal, equivocal, abnormal with fixed defects, abnormal with fully reversible defects, or abnormal with partially reversible defects. MPI images were processed using recently developed analysis software (AutoSPECT [CedarsSinai Medical Center] and QPS AutoQUANT). Briefly, this commercial package consists of three software modules that determine reconstruction limits for the projection dataset, reconstruct the projection images into transaxial images using standard filtered back projection, and then reorient the transaxial images into short-axis images. Quantitative defect extent and severity are defined from sex-specific normal limits, and an SSS is obtained by adding the scores of the 20 segments $(0=$ normal to $4=$ absent uptake) of the stress sestamibi images. The software processing and quantitative analysis were performed without reference to the initial visual interpretation or knowledge of the patient outcomes. ${ }^{10}$ An SSS $<4$ was considered normal for the quantitative assessment, whereas an SSS $\geq 4$ was taken as abnormal. ${ }^{11}$ Abnormal scans were classified as mildly abnormal (4-8), moderately abnormal (9-13), and severely abnormal (>13). ${ }^{12}$ In 29 patients with abnormal MPI, quantitative coronary angiography was performed by standard techniques.

\section{Statistical Analysis}

Mean \pm SD and proportions were used to summarize the characteristics of the study sample. Continuous variables were compared by ANOVA, and categorical variables were compared by the Kruskal-Wallis non-parametric ANOVA. Linear and logistic regression analyses were employed to assess the relationship of extent of myocardial perfusion defect (MPD) and TR with and without adjustment for conventional cardiovascular risk factors. ROC curves were constructed and the area under the curve (AUC) was calculated to predict the ability of each model to detect abnormal MPI (SSS $\geq 4$ ).

Variability and repeatability of the test were measured and compared between repeated DTM measurements. Assessment of variability included the calculation of the coefficient of variation $(\mathrm{CV})$ of the TR and AUC for repeated DTM measurements, day-to-day variability of the participants, intra-class correlation coefficient (ICC) in a two-way mixed effects model, and Bland \& Altman plots with calculation of the repeatability coefficient. All statistical analyses were performed with STATA version 8.0 (Stata Corp., Austin, Texas, http://www.stata. com). The level of significance was set at $P<.05$ (two-tailed). The study protocol and consent form were approved by IRB Committee Board of Los Angeles Biomedical Research Institute at Harbor UCLA Medical Center, Torrance, CA.

\section{RESULTS}

Characteristics of the study population are highlighted in Table 1 according to the extent of MPD among the 116 consecutive participants (age $57 \pm 10$ years, 64 (55\%) male). Thirty-eight $(33 \%)$ had abnormal MPI (13 with mild MPD ( $4 \leq \mathrm{SSS} \leq 8)$, 13 with moderate MPD $(9 \leq \mathrm{SSS} \leq 13)$, and 12 with severe MPD (SSS > 13). Twenty-nine patients with abnormal MPI underwent quantitative coronary angiography (QCA) and 27 (93\%) had obstructive CAD defined by $>50 \%$ luminal stenosis. There were no significant statistical differences between normal and abnormal MPI cohorts in age, hypertension, hypercholesterolemia, diabetes, smoking status, and family history of premature CHD, left ventricular ejection fraction (LVEF), total cholesterol, LDL-C and triglyceride. FRS increased significantly with increasing the extent of MPD (Table 1).

\section{Vascular Function and Myocardial Perfusion}

The prevalence of MPD according to tertiles of TR is demonstrated in Figure 2A. TR and AUC reduced significantly with increasing the extent of MPD (Table 2) and with diabetes mellitus (Figure 2B) $(P<.05)$. TR was inversely correlated with increasing the extent of MPD ( $\mathrm{r}=-0.88, P=.0001)$ (Figure 3 ) and reduced significantly from SSS $<4$ to $4 \leq \mathrm{SSS} \leq 8$ to $9 \leq \mathrm{SSS} \leq 13$ to $\mathrm{SSS}>13(P=.0001)$ (Table 1$)$.

The independent association of TR with the extent of MPD is demonstrated in a multivariate logistic regression analysis (Table 3 ). After adjustment for age, gender, and conventional cardiovascular risk factors the 
Table 1. Cardiovascular risk factors and the extent of myocardial perfusion defect

\begin{tabular}{|c|c|c|c|c|c|}
\hline & $\begin{array}{c}\text { Normal MPD } \\
\text { SSS }<4 \\
N=78\end{array}$ & $\begin{array}{c}\text { Mild MPD } \\
4 \leq \text { SSS } \leq 8 \\
N=13\end{array}$ & $\begin{array}{c}\text { Moderate MPD } \\
9 \leq \text { SSS } \leq 13 \\
N=13\end{array}$ & $\begin{array}{c}\text { Severe MPD } \\
\text { SSS >13 } \\
N=12\end{array}$ & $P$ value \\
\hline Age (years) & $56 \pm 10$ & $56 \pm 8$ & $57 \pm 12$ & $56 \pm 9$ & .98 \\
\hline Female gender & $60 \%$ & $46 \%$ & $38 \%$ & $50 \%$ & .42 \\
\hline Smoking & $15.8 \%$ & $26.5 \%$ & $15.5 \%$ & $26 \%$ & .14 \\
\hline $\mathrm{SPB}(\mathrm{mmHg})$ & $137 \pm 23$ & $135 \pm 20$ & $140 \pm 24$ & $136 \pm 24$ & .09 \\
\hline $\mathrm{DBP}(\mathrm{mmHg})$ & $76 \pm 13$ & $74 \pm 11$ & $82 \pm 10$ & $76 \pm 14$ & .36 \\
\hline Hypertension $^{\dagger}$ & $84 \%$ & $85 \%$ & $92 \%$ & $96 \%$ & .52 \\
\hline Anti-hypertensive Medication & $87 \%$ & $88 \%$ & $92 \%$ & $100 \%$ & .57 \\
\hline Total Cholesterol (mg/dL) & $176 \pm 49$ & $174 \pm 49$ & $161 \pm 42$ & $182 \pm 50$ & .79 \\
\hline $\mathrm{HDL}-\mathrm{C}(\mathrm{mg} / \mathrm{dL})$ & $40 \pm 12$ & $38 \pm 11$ & $36 \pm 11$ & $34 \pm 11$ & .02 \\
\hline LDL-C (mg/dL) & $110 \pm 42$ & $104 \pm 37$ & $101 \pm 31$ & $98 \pm 39$ & .71 \\
\hline Triglycerides $(\mathrm{mg} / \mathrm{dL})$ & $138 \pm 71$ & $175 \pm 113$ & $124 \pm 91$ & $284 \pm 118$ & .01 \\
\hline High cholesterol $^{\ddagger}$ & $55 \%$ & $77 \%$ & $54 \%$ & $67 \%$ & .46 \\
\hline Cholesterol-lowering medication & $89 \%$ & $84 \%$ & $95 \%$ & $95 \%$ & .61 \\
\hline Diabetes mellitus ${ }^{\S}$ & $36 \%$ & $46 \%$ & $54 \%$ & $75 \%$ & .06 \\
\hline Family history of $\mathrm{CHD}^{\prime \prime}$ & $50 \%$ & $52 \%$ & $50 \%$ & $50 \%$ & .93 \\
\hline Body mass index $(\mathrm{kg} / \mathrm{m} 2)$ & $31.5 \pm 8.1$ & $31.8 \pm 8.6$ & $30.4 \pm 7.9$ & $31.9 \pm 9.1$ & .96 \\
\hline Framingham Risk Score (\%) & $10.6 \pm 4.9$ & $14.7 \pm 12.8$ & $17.1 \pm 9.8$ & $17.2 \pm 7.6$ & .002 \\
\hline $\mathrm{LV} \mathrm{EF}^{\diamond}$ & $57.8 \pm 2.1$ & $58.1 \pm 2.8$ & $55.6 \pm 3.2$ & $57.5 \pm 3.7$ & .61 \\
\hline Known history of CAD & $7 \pm 3$ & $8 \pm 2$ & $8 \pm 2$ & $8 \pm 2$ & .75 \\
\hline Temperature rebound (TR) & $1.61 \pm 0.15$ & $0.51 \pm 0.22$ & $0.26 \pm 0.15$ & $-0.37 \pm 0.19$ & .0001 \\
\hline Area under the curve (AUC) & $383 \pm 48$ & $143 \pm 30$ & $96 \pm 43$ & $39 \pm 17$ & .0001 \\
\hline
\end{tabular}

Values presented as mean \pm SD or $\%$.

$N S$, Non-significant $(P>.05)$.

$\dagger$ Self-reported diagnosis of hypertension, prescribed medication for hypertension, or current blood pressure $>140 \mathrm{mmHg}$ systolic or $>90 \mathrm{mmHg}$ diastolic (>130/80 $\mathrm{mmHg}$ if diabetic).

$¥$ Self-reported diagnosis of high cholesterol, prescribed medication for high cholesterol, or current total cholesterol $>200 \mathrm{mg} / \mathrm{dL}$.

$\S$ Self-reported diagnosis of diabetes (type 1 or 2 ) or prescribed medication for diabetes.

II First degree relative; female $<65$ years, male $<55$ years.

$\diamond$ Left ventricular ejection fraction on G-SPECT.
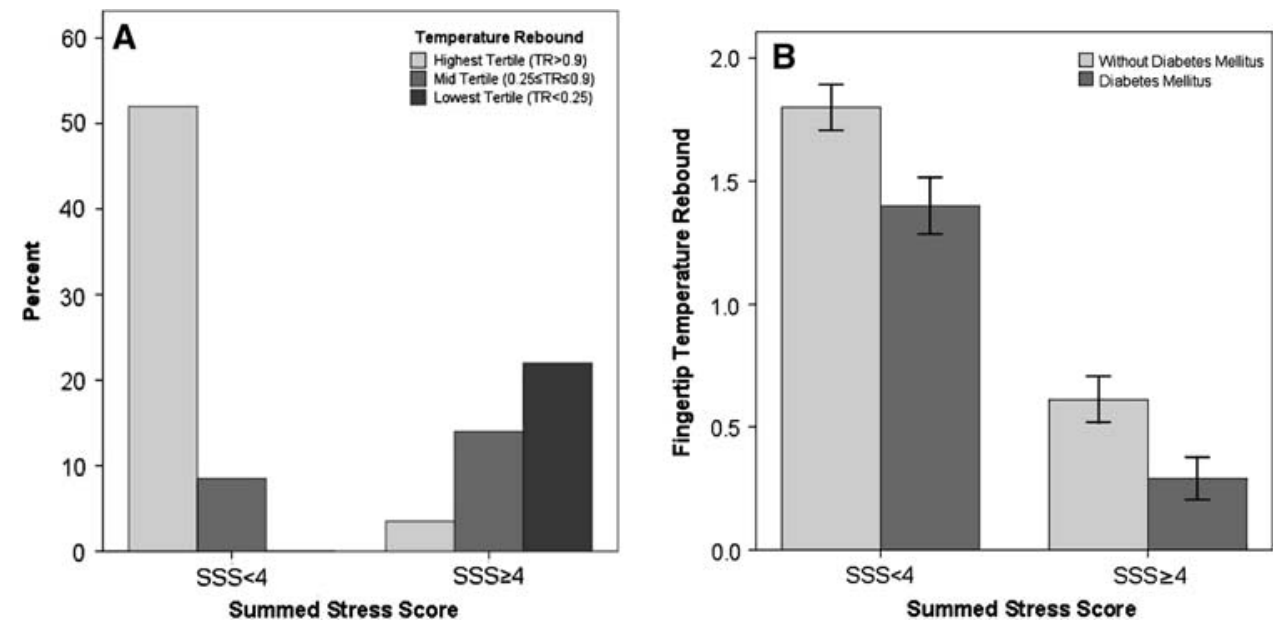

Figure 2. A, Myocardial perfusion imaging abnormalities (\%) in TR tertiles. B, Vascular dysfunction increased proportionally with diabetes mellitus and the extent of myocardial perfusion defect. 
Table 2. Linear regression analysis of the relationship between the extent of myocardial perfusion defect and vascular dysfunction $(\beta, 95 \% \mathrm{Cl})$

\begin{tabular}{lccc}
\hline Model SSS $<\mathbf{4}$ & $\mathbf{4} \leq$ SSS $\leq \mathbf{8}$ & $\mathbf{9} \leq$ SSS $\leq \mathbf{1 3}$ & SSS $>13$ \\
\hline \multicolumn{2}{l}{ Unadjusted } \\
TR 1 (ref) & -0.34 & -0.82 & -0.96 \\
& $(-0.91$ to -0.23$), P=.002$ & $(-1.22$ to -0.43$), P=.0001$ & $(-1.26$ to -0.67$), P=.0001$
\end{tabular}

Adjusted for age, gender, diabetes mellitus, hypertension, hypercholesterolemia, family history of CHD, and smoking status
TR
1 (ref)
$-0.35$
$-0.84$
$-1.03$
$(-0.92$ to -0.25$), P=.003 \quad(-1.25$ to -0.44$), P=.0001 \quad(-1.34$ to -0.71$), P=.0001$

$T R$, Temperature rebound; $C I$, confidence interval.
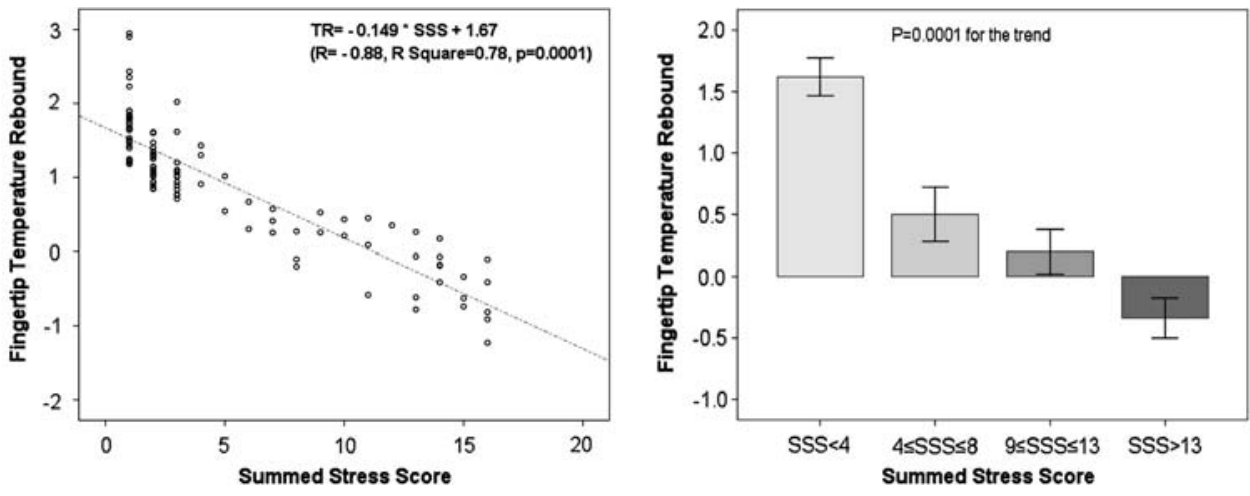

Figure 3. Vascular dysfunction and the extent of myocardial perfusion defect.

odds ratio of lowest versus two upper tertiles of TR was 3.93 (95\% CI: $1.66-9.29, P=.002$ ) for $\mathrm{SSS} \geq 4$ and $9.65(95 \%$ CI $3.19-10.15, P=.0001)$ for $\mathrm{SSS} \geq 8$ compared to SSS $<4$.

ROC curves were constructed to assess the ability of a combination of clinical variables to predict $\mathrm{SSS} \geq 4$. The area under the curve (AUC) for three proposed models is summarized in Figure 4. TR was superior to the FRS $(\mathrm{AUC} \pm \mathrm{SD} \quad 0.75 \pm 0.04$ vs $0.65 \pm 0.05$,
$P=.04)$. The addition of TR to FRS significantly improved the AUC compared to FRS alone (AUC \pm SD $0.84 \pm 0.03$ vs $0.65 \pm 0.05, P=.001)$ and provided a largest contribution to predict $\mathrm{SSS}>4$.

\section{Repeated DTM Measurements}

Day to day intra-subject variability was $6.2 \%$ for baseline temperature, $8.7 \%$ for mean blood pressure, and

Table 3. Logistic regression analysis of the relationship between significant myocardial perfusion defect and increased vascular dysfunction

\begin{tabular}{|c|c|c|c|}
\hline Model & SSS $<4$ & SSS $\geq 4$ & SSS $\geq 8$ \\
\hline \multicolumn{4}{|l|}{ Unadjusted } \\
\hline Odds of $\mathrm{TR}^{¥}$ & 1 (ref) & $4.23(1.83-9.78), P=.001$ & $9.67(3.33-9.78), P=.0001$ \\
\hline \multicolumn{4}{|c|}{ Adjusted for age, gender, diabetes mellitus, hypertension, hypercholesterolemia, family history of CHD and smoking status } \\
\hline Odds $\mathrm{TR}^{¥}$ & 1 (ref) & $3.93(1.66-9.29), P=.002$ & 9.65 (3.19-10.15), $P=.0001$ \\
\hline
\end{tabular}

$T R$, Temperature rebound.

$¥$ Odds of lowest versus two upper tertile of TR $(T R \leq 0.25$ vs $T R>0.25)$. 


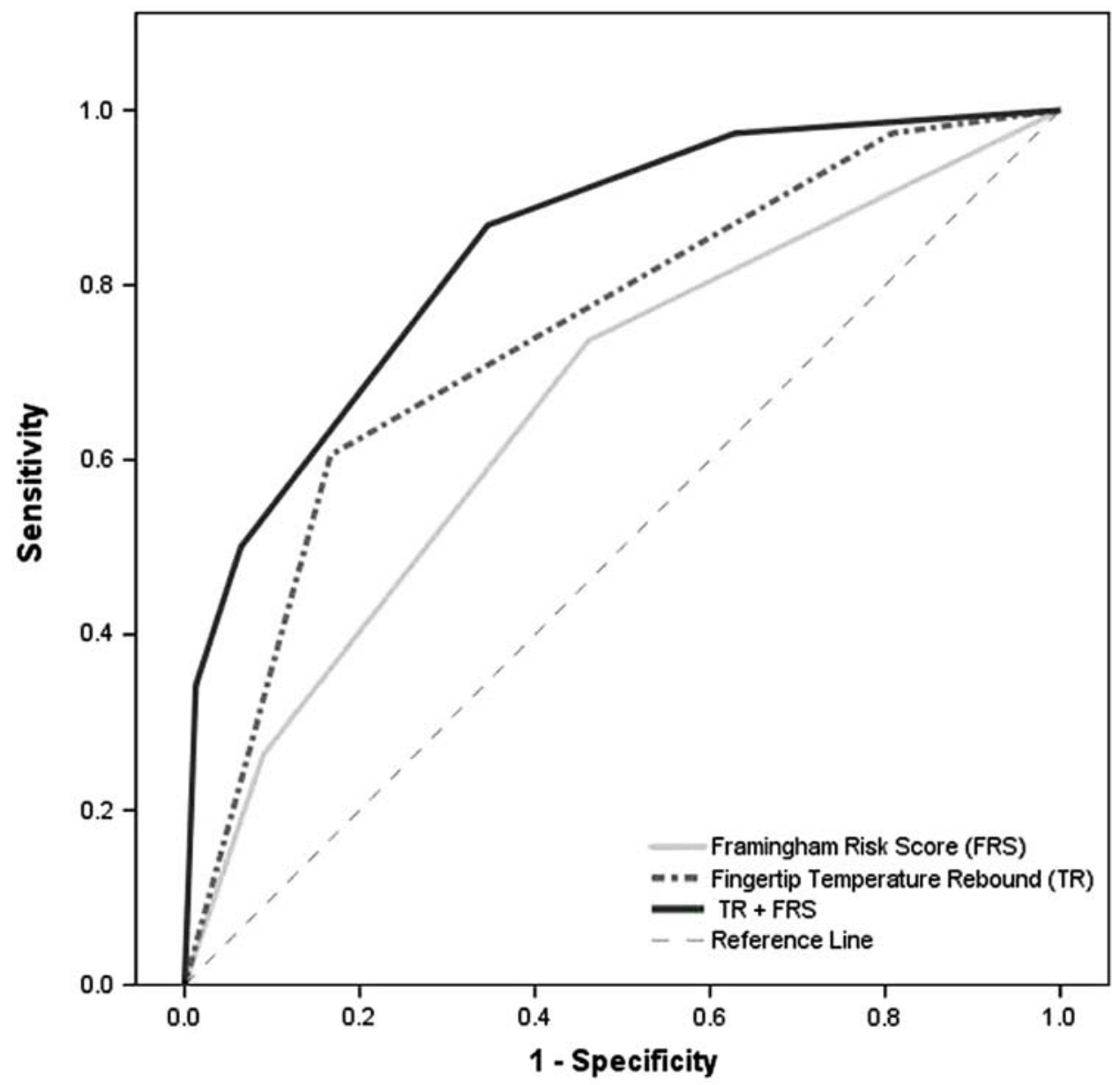

\begin{tabular}{ccccc}
\hline Variable & AUC \pm SD & $95 \% \mathbf{C l}$ & $\mathbf{P}$ & Comparison P with FRS \\
\hline FRS $^{*}$ & $0.65 \pm 0.05$ & $0.56-0.77$ & 0.004 & -- \\
\hline TR $^{\epsilon}$ & $0.75 \pm 0.04$ & $0.65-0.84$ & 0.0001 & 0.04 \\
\hline FRS+TR & $0.84 \pm 0.03$ & $0.77-0.91$ & 0.0001 & 0.001
\end{tabular}

Framingham 10 year CHD risk Score (FRS): $<10 \%, 10-20 \%$ and $>20 \%$

$€$ Temperature Rebound: Tertiles of TR

Figure 4. ROC curves to assess the diagnostic accuracy of TR and clinical variables for the detection of abnormal myocardial perfusion ( $\mathrm{SSS} \geq 4$ ) in symptomatic patients.

$11.4 \%$ for heart rate. The coefficient of repeatability (CR) of TR and AUC were $2.4 \%$ and $2.8 \%$, respectively. The intra-class correlation coefficient of repeated DTM measurements was 0.82 for TR and 0.83 for AUC $(P=.0001)$ (Figure 5).

\section{DISCUSSION}

The data clearly demonstrate the strong relationship of vascular dysfunction measured by DTM and the extent of MPD independent of age, gender, and conventional cardiac risk factors. These results extend our previous observations that vascular dysfunction measured by DTM is associated with both underlying clinical and subclinical coronary artery disease. ${ }^{6}$

\section{Vascular Function and Myocardial Perfusion}

It is well known that a relationship between vascular dysfunction and coronary risk factors exists. ${ }^{13-15}$ Nevertheless, there is a considerable heterogeneity in the 


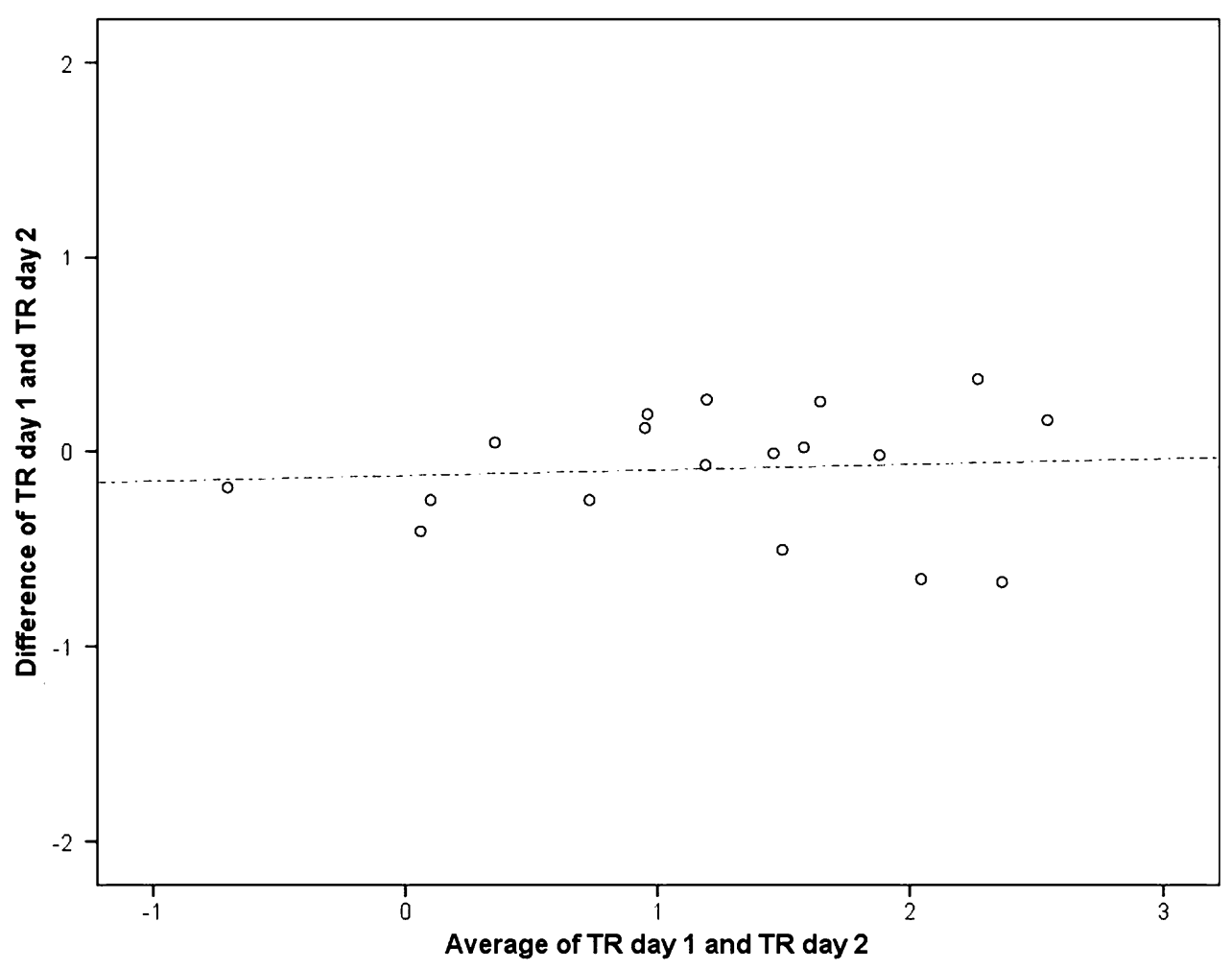

\begin{tabular}{|c|c|c|c|c|c|c|}
\hline Variable & D & $S D_{D}$ & $\mathrm{CV}(\%)$ & CR $(\%)$ & $\mathrm{ICC}$ & $P$ value \\
\hline Heart Rate & 0.47 & 0.054 & 11.4 & 10.6 & 0.70 & 0.01 \\
\hline MAP & 0.44 & 0.038 & 8.7 & 7.5 & 0.79 & 0.0005 \\
\hline TMP initial & 0.51 & 0.036 & 7.1 & 7.1 & 0.81 & 0.0001 \\
\hline \multicolumn{7}{|c|}{ DTM Indices of vascular reactivity } \\
\hline $\mathrm{TR}^{\circ} \mathrm{C}$ & 0.209 & 0.012 & 5.7 & 2.4 & 0.82 & 0.0001 \\
\hline$A \cup C$ & 0.292 & 0.014 & 4.8 & 2.8 & 0.83 & 0.0001 \\
\hline
\end{tabular}

Figure 5. Coefficient of repeatability and variability of repeated DTM measurements.

magnitude of vascular dysfunction in individuals with similar risk factor profile. ${ }^{16,17}$ In this regard, vascular dysfunction may be seen as an important "integrative factor" of the inherent atherosclerotic risk in an individual, taking into account the cumulative risk of various coronary risk factors, unknown variables, and the genetic susceptibility of vascular endothelium toward coronary risk factors. ${ }^{18,19}$ This integrative role of vascular dysfunction may explain the additive value of vascular function to predict the occurrence stress- induced perfusion defects apart from the Framingham Risk Score. $^{20-23}$

Vascular dysfunction is a characteristic feature throughout the development of atherosclerosis, and is independently related to adverse cardiovascular risk. ${ }^{24,25}$ Gould and Lipscomb ${ }^{26}$ first demonstrated that hyperemic coronary blood flow was attenuated in the presence of stenoses that exceeded $50 \%$ of the coronary luminal diameter. Wie et $\mathrm{al}^{27}$ demonstrated that reversible defects on ${ }^{99 \mathrm{~m}} \mathrm{Tc}$-sestamibi during vasodilator stress 
imaging are related to decreases in myocardial blood volume (MBV) distal to a stenosis and not to "flow mismatch", between beds and the decrease in MBV results in reduced ${ }^{99 \mathrm{~m}}$ Tc-sestamibi uptake during hyperemia. Other studies have demonstrated that areas with a perfusion abnormality on stress SPECT were associated with impaired coronary flow reserve (CFR). ${ }^{24}$ Similarly, peripheral vascular function measured through reactive hyperemia procedures have correlated with coronary flow reserve in response to acetylcholine. $^{25}$ In the same fashion, flow-mediated brachial artery dilation during and after reactive hyperemia was predictive of the presence and extent of abnormal myocardial perfusion reserve. ${ }^{28-30}$ The present study confirms and extends these findings by demonstrating that vascular dysfunction measured by DTM is associated with the extent of MPD in which the risk of significant MPD was at least nine times higher in subjects with severe vascular dysfunction. Addition of TR to FRS provided the greatest diagnostic power for predicting abnormal MPI.

\section{CLINICAL IMPLICATIONS}

The increased appreciation of vascular dysfunction as a marker for cardiovascular disease has resulted in the development of non-invasive tools designed for both initial risk assessment and the evaluation of changes in vascular status over time. The strong association between vascular dysfunction measured by DTM with the extent of MPD reconfirms our previous findings which have shown that vascular dysfunction measured by DTM correlates well with the FRS, insulin resistance, metabolic syndrome and diabetes, and the extent of coronary artery disease measured by coronary artery calcium (CAC) and computed tomographic angiography(CTA $)^{31-35}$ and suggest a role for this new technique in the evaluation of asymptomatic at risk patients.

\section{LIMITATIONS}

The present study has several limitations. First, only symptomatic patients who underwent myocardial perfusion imaging were included. Abnormalities noted by scintigraphy may not always correspond to angiographically significant $\mathrm{CAD}$ due to physiologic, technical, and population based factors. ${ }^{36}$ However among 29 patients who underwent quantitative coronary angiography (QCA), 27 (93\%) had obstructive CAD ( $>50 \%$ luminal stenosis). Second, despite the limitation in the selection of patients who most likely have some degree of vascular dysfunction, hypertension, and diabetes mellitus, stepwise regression analysis showed that the association between MPD and TR is independent of conventional cardiac risk factors. Third, it is widely known that even severe vascular dysfunction may be present even in the absence of flow-limiting epicardial lesions. However, our study demonstrated that patients with severe vascular dysfunction and increased FRS had higher likelihood of MPD.

\section{CONCLUSION}

Vascular dysfunction measured by DTM is associated with the extent of myocardial perfusion defect measured by G-SPECT independent of age, gender, and conventional cardiovascular risk factors. Addition of vascular dysfunction measured by DTM to Framingham risk score provides incremental value for identification of patients with abnormal myocardial perfusion. Prospective studies are needed to determine the clinical utility and cost effectiveness of incorporating of DTM in risk assessment of patients suspected for coronary heart disease.

\section{Open Access}

This article is distributed under the terms of the Creative Commons Attribution Noncommercial License which permits any noncommercial use, distribution, and reproduction in any medium, provided the original author(s) and source are credited.

\section{References}

1. Drexler H, Hornig B. Endothelial dysfunction in human disease. J Mol Cell Cardiol 1999;31:51-60.

2. Kvernmo K, Slagsvold CE, Gjolberg T. Laser Doppler flux reappearance time (FRT) in patients with lower limb atherosclerosis and healthy controls. Eur J Vasc Surg 1998;2:171-6.

3. Matsubara J, Namuri J, Nagasue M, Sakamoto S, Yuasa K, Shimizu T. Postocclusive reactive hyperemia during vascular reconstruction. Int J Angiol 1998;3:222-7.

4. Wahlberg E, Olofson P, Takolander R, Swedenborg J. Postocclusive reactive hyperemia estimating peripheral vascular resistance: A non invasive method to predict outcome of infrainguinal vascular reconstructions? J Cardiovasc Surg (Torino) 1996;37:545-52.

5. Wilkin JK. Periodic cutaneous blood flow during postocclusive reactive hyperemia. Am J Physiol 1986;250:H765-8.

6. Ahmadi N, Hajsadeghi F, Gul K, Vane J, Usman N, Flores F, Nasir K, Hecht H, Naghavi M, Budoff M. Relations between digital thermal monitoring of vascular function, the Framingham risk score, and coronary artery calcium score. J Cardiovasc Comput Tomogr 2008;2(6):382-8.

7. Dhindsa M, Sommerlad SM, De Van AE, Barnes JN, Sugawara J, Ley $\mathrm{O}$, et al. Interrelationships among noninvasive measures of postischemic macro- and microvascular reactivity. J Appl Physiol 2008;105:427-32.

8. Wilson PWF, D'Agostino RB, Levy D, Belanger AM, Silbershatz H, Kannel WB. Prediction of coronary heart disease using risk factor categories. Circulation 1998;97:1837-47. 
9. Berman DS, Abidov A, Kang X, Hayes SW, Friedman JD, Sciammarella MG, et al. Prognostic validation of a 17-segment score derived from a 20-segment score for myocardial perfusion SPECT interpretation. J Nucl Cardiol 2004;11:414-23.

10. Berman DS, Kang X, Hayes SW, Friedman JD, Cohen I, Abidov A, et al. Adenosine myocardial perfusion single-photon emission computed tomography in women compared with men. Impact of diabetes mellitus on incremental prognostic value and effect on patient management. J Am Coll Cardiol 2003;41:1125-33.

11. Hachamovitch R, Berman DS, Shaw LJ, Kiat H, Cohen I, Cabico $\mathrm{JA}$, et al. Incremental prognostic value of myocardial perfusion single photon emission computed tomography for the prediction of cardiac death: Differential stratification for risk of cardiac death and myocardial infarction. Circulation 1998;97:535-43.

12. Sharir T, Germano G, Kang X, Lewin HC, Miranda R, Cohen I, et al. Prediction of myocardial infarction versus cardiac death by gated myocardial perfusion SPECT: Risk stratification by the amount of stress-induced ischemia and the poststress ejection fraction. J Nucl Med 2001;42:831-7.

13. Bulut D, Maier K, Bulut-Streich N, Borgel J, Hanefeld C, Mugge A. Circulating endothelial microparticles correlate inversely with endothelial function in patients with ischemic left ventricular dysfunction. J Card Fail 2008;14:336-40.

14. Weintraub HS. Identifying the vulnerable patient with ruptureprone plaque. Am J Cardiol 2008;101:3F-10F.

15. Kubrich M, Petrakopoulou P, Kofler S, Nickel T, Kaczmarek I, Meiser BM, et al. Impact of coronary endothelial dysfunction on adverse long-term outcome after heart transplantation. Transplantation 2008;85:1580-7.

16. Marroquin OC, Holubkov R, Edmundowicz D, Rickens C, Pohost $\mathrm{G}$, Buchthal S, et al. Heterogeneity of microvascular dysfunction in women with chest pain not attributable to coronary artery disease: Implications for clinical practice. Am Heart J 2003;145:628-35.

17. Schindler TH, Facta AD, Prior JO, Cadenas J, Zhang XL, Li Y, et al. Structural alterations of the coronary arterial wall are associated with myocardial flow heterogeneity in type 2 diabetes mellitus. Eur J Nucl Med Mol Imaging 2008. doi:10.1007/s00259008-0885-z.

18. Shechter M, Issachar A, Marai I, Koren-Morag N, Freinark D, Shahar Y, Feinberg MS et al. Long-term association of brachial artery flow-mediated vasodilation and cardiovascular events in middle-aged subjects with no apparent heart disease. Int J Cardiol 2008.

19. Halcox JP, Schenke WH, Zalos G, Mincemoyer R, Prasad A, Waclawiw MA, et al. Prognostic value of coronary vascular endothelial dysfunction. Circulation 2002;106:653-8.

20. Chironi G, Craiem D, Miranda-Lacet J, Levenson J, Simon A. Impact of shear stimulus, risk factor burden and early atherosclerosis on the time-course of brachial artery flow-mediated vasodilation. J Hypertens 2008;26:508-15.

21. Lau KK, Chan YH, Yiu KH, Tam S, Li SW, Lau CP, et al. Incremental predictive value of vascular assessments combined with the Framingham Risk Score for prediction of coronary events in subjects of low-intermediate risk. Postgrad Med J 2008;84:153-7.

22. Wang L, Jerosch-Herold M, Jacobs DR Jr, Shahar E, Folsom AR. Coronary risk factors and myocardial perfusion in asymptomatic adults: The Multi-Ethnic Study of Atherosclerosis (MESA). J Am Coll Cardiol 2006;47:565-72.

23. Dorbala S, Hassan A, Heinonen T, Schelbert HR, Di Carli MF. Coronary vasodilator reserve and Framingham risk scores in subjects at risk for coronary artery disease. J Nucl Cardiol 2006;13:761-7.

24. Yoshinaga K, Katoh C, Noriyasu K, Iwado Y, Furuyama H, Ito Y, et al. Reduction of coronary flow reserve in areas with and without ischemia on stress perfusion imaging in patients with coronary artery disease: A study using oxygen 15-labeled water PET. J Nucl Cardiol 2003; 10:275-83.

25. Bonetti PO, Pumper GM, Higano ST, Holmes DR Jr, Kuvin JT, Lerman A. Noninvasive identification of patients with early coronary atherosclerosis by assessment of digital reactive hyperemia. J Am Coll Cardiol 2004;44:2137-41.

26. Gould KL, Lipscomb K. Effects of coronary stenoses on coronary flow reserve and resistance. Am J Cardiol 1974;34:48-55.

27. Wei K, Firoozan S, Jayaweera AR, Skyba DM, Linka A, Kaul S. Quantification of myocardial blood flow with ultrasound-induced destruction of microbubbles administered as a constant venous infusion. Circulation 1998;97:473-8.

28. Wu WC, Sharma SC, Choudhary G, Coulter L, Coccio E, Eaton CB. Flow-mediated vasodilation predicts the presence and extent of coronary artery disease assessed by stress thallium imaging. J Nucl Cardiol 2005;12:538-44.

29. Kuvin JT, Patel AR, Sliney KA, Pandian NG, Rand WM, Udelson JE, et al. Peripheral vascular endothelial function testing as a noninvasive indicator of coronary artery disease. J Am Coll Cardiol 2001;38:1843-9.

30. Perrone-Filardi P, Cuocolo A, Brevetti G, Silvestro A, Storto G, Dellegrottaglie $S$, et al. Relation of brachial artery flow-mediated vasodilation to significant coronary artery disease in patients with peripheral arterial disease. Am J Cardiol 2005;96:1337-41.

31. Ahmadi N, Nuguri V, Osman N, Hajsadeghi F, Honoris L, Gul K, et al. Digital thermal reactivity decreases with the severity of coronary artery disease diagnosed by 64-multiderectors. J Am Coll Cardiol 2008;51:A98-177.

32. Ahmadi N, Gul K, Hajsadeghi F, Jamieson C, Gopal A, Naghavi M, et al. Fingertip thermal reactivity correlated with Framingham Risk Score. J Cardiovasc Comput Tomogr 2007;1:S13.

33. Ahmadi N, Hajsadeghi F, Gul K, Lee R, Vane J, Chau G, et al. Vascular function measured by fingertip thermal reactivity is impaired in patients with insulin resistance. Circulation 2008; 117:e198-291

34. Ahmadi N, Hajsadeghi F, Gul K, Usman N, Vane J, Jamieson C, et al. Digital thermal monitoring of vascular function is associated with coronary artery calcification in asymptomatic adults. Circulation 2008;117:e409-68.

35. Ahmadi N, Hajsadeghi F, Gul K, Leibfried M, DeMoss D, Nasir $\mathrm{K}$, et al. Vascular function measured by fingertip thermal reactivity is impaired in patients with metabolic syndrome and diabetes mellitus. Arterioscler Thromb Vasc Biol 2008;28:e32-149.

36. Ammann P, Naegeli B, Rickli H, Buchholz S, Mury R, Schuiki E, et al. Characteristics of patients with abnormal stress technetium Tc 99m sestamibi SPECT studies without significant coronary artery diameter stenoses. Clin Cardiol 2003;26:521-4. 